6. Freedland SJ, Partin AW, Epstein JI, Walsh PC: Biochemical failure after radical prostatectomy in men with pathologic organ-confined disease: pT2a versus pT2b. Cancer. 2004; 100: 1646-9.

7. Chun FK, Briganti A, Lebeau T, Fradet V, Steuber T, Walz J, et al.: The 2002 AJCC pT2 substages confer no prognostic information on the rate of biochemical recurrence after radical prostatectomy. Eur Urol. 2006; 49: 273-8; discussion 278-9.

8. Hong SK, Han BK, Chung JS, Park DS, Jeong SJ, Byun SS, et al.: Evaluation of pT2 subdivisions in the TNM staging system for prostate cancer. BJU Int. 2008; 102: 1092-6.

Dr. Athanase Billis

Full-Professor of Pathology State University of Campinas, Unicamp

Campinas, São Paulo, Brazil

E-mail: athanase@fcm.unicamp.br

doi: 10.1590/S1677-553820100002000021

\title{
Radical prostatectomy (RP) findings in cases with only intraductal carcinoma of the prostate (IDC-P) on needle biopsy
}

Robinson BD, Epstein JI

Dep. of Pathology, The Johns Hopkins Hospital, Baltimore, MD, USA

Mod Pathol 2010; 23 (suppl 1): 215A

Background: When IDC-P is present on biopsy, it is usually seen with infiltrating acinar adenocarcinoma. In 2006, we reported 27 cases with IDC-P only on biopsy; however, only 6 cases had available RP findings.

Design: 82 men with IDC-P only on prostate biopsy were identified from the consult files of one of the authors. Follow-up information was available in 66 cases. 20 men were treated with RP, 17 radiation therapy (RT), 8 hormone therapy (HT), 13 RT and HT, 6 active surveillance, and 2 rebiopsy. An attempt was made to retrieve the slides of all $20 \mathrm{RP}$ cases.

Results: Of the 20 RP cases, 5 showed extraprostatic extension, 3 seminal vesicle invasion, 10 were organ-confined, and 2 showed extensive IDC-P only without identifiable invasive cancer. Of the 18 cases with invasive cancer, the average Gleason score (GS) was 7.8. 1 patient developed bone metastases 3 years post-RP, and 3 others were post-RP PSA failures. 13 RPs were available for our review. 9 showed extensive IDC-P (including one case of IDC-P only), defined as $>10 \%$ of the tumor volume being intraductal; 3 focal IDC-P; and 1 no IDC-P. All cases with invasive carcinoma were acinar, although 3 cases were classified as ductal by referring pathologists. We concurred with the outside GS in 5/13 cases ( 5 undergraded, 3 overgraded). In the 3 cases that we gave lower GS, the outside institution graded cribiform IDC-P with and without necrosis as Gleason pattern 5 or 4 , respectively.

Conclusions: Our study, the largest to date with RP findings following IDC-P only on needle biopsy, confirms that aggressive therapy is appropriate for patients whose biopsies show only IDC-P. It is likely that the pathological findings are even worse than we report herein, as most RPs were only partially sampled. Most cases likely represent intraductal spread of high grade cancer, but some cases represent in situ acinar adenocarcinoma.

\section{Editorial Comment}

Intraductal carcinoma of the prostate (IDC-P) is defined as presence of atypical cells that span the entire lumen of prostatic ducts or acini while the normal architecture of ducts or acini is still maintained including presence of basal cells $(1,2)$. With presence of IDC-P on a biopsy, the pathologist considers 4 possibilities: 1 ) 
ductal carcinoma; 2) high-grade intraepithelial neoplasia (HGPIN); 3) intraductal carcinoma; and, 4) intraductal spread of an invasive carcinoma.

Ductal carcinoma may be ruled out because of the presence of basal cells; in cases of irregularity and distortion of the ducts, HGPIN may also be ruled out; and, in presence of an invasive carcinoma, intraductal spread is most probable and the finding adds no additional information to the report. The problem is related to cases that IDC-P is the only finding on a biopsy.

The Johns Hopkins group studied 20 radical prostatectomy specimens of patients who presented on biopsy only the diagnosis of IDC-P. Two out of twenty patients showed extensive IDC-P only, without identifiable invasive cancer. IDC-P in these 2 patients may represent: 1 . an early phase of ductal or acinar carcinoma; or 2. intraductal spread of an invasive not detected tumor.

According to the authors, IDC-P as the only finding on needle biopsy corresponds to almost always to aggressive tumors and the patients may have definitive treatment. A more conservative approach would be an extended rebiopsy in order to detect a possible invasive cancer.

\title{
References
}

1. Cohen RJ, McNeal JE, Baillie T: Patterns of differentiation and proliferation in intraductal carcinoma of the prostate: significance for cancer progression. Prostate. 2000; 43: 11-9.

2. Guo CC, Epstein JI: Intraductal carcinoma of the prostate on needle biopsy: Histologic features and clinical significance. Mod Pathol. 2006; 19: 1528-35.

\author{
Dr. Athanase Billis \\ Full-Professor of Pathology \\ State University of Campinas, Unicamp \\ Campinas, São Paulo, Brazil \\ E-mail: athanase@fcm.unicamp.br
}

\section{BASIC AND TRANSLATIONAL UROLOGY}

\section{Potential mechanism of action of human growth hormone on isolated human penile erectile tissue}

Uckert S, Scheller F, Stief CG, Knapp WH, Sohn M, Becker AJ, Kuczyk MA

Department of Urology and Uro-Oncology, Hannover Medical School, Hannover, Germany

Urology. 2010; 75: 968-73

Objectives: To evaluate the mechanisms of growth hormone $(\mathrm{GH})$ action on isolated human penile erectile tissue. Human $\mathrm{GH}(\mathrm{hGH})$ has been suggested to play a role in male reproductive function, including penile erection. Nevertheless, it still remains unclear which intracellular pathways mediate the physiological effects of $\mathrm{GH}$ on the human corpus cavernosum (HCC).

Methods: Using the organ bath technique, the effects of GH were investigated on electrical field stimulation (EFS)-induced relaxation of isolated $\mathrm{HCC}$ in the absence and presence of the guanylyl cyclase inhibitor $1 \mathrm{H}-$ 